\title{
Accelerator systems for the International Design Study of the Neutrino Factory
}

\author{
F.J.P. Soler ${ }^{* \dagger}$ \\ School of Physics and Astronomy, University of Glasgow, Glasgow, G12 8QQ, UK. \\ E-mail: paul.soler@glasgow.ac.uk
}

The Neutrino Factory produces high-energy neutrino beams with a well-defined flavour content and energy spectrum from the decay of intense, high-energy, stored muon beams. The muon storage rings include long straight sections that are directed toward neutrino detectors that are sited several thousand kilometers away. This paper outlines the status of the accelerator facility described in the Interim Design Report (IDR) recently completed by the International Design Study for a Neutrino Factory (IDS-NF). We give a baseline specification for the accelerator, describe the accelerator subsystems that comprise it and briefly indicate some of the accelerator-physics challenges that such a facility presents.

The 2011 Europhysics Conference on High Energy Physics, EPS-HEP 2011,

July 21-27, 2011

Grenoble, Rhône-Alpes, France

*Speaker.

$\dagger$ On behalf of the IDS-NF Collaboration. 


\section{Introduction}

The Neutrino Factory is a facility in which a neutrino beam is created from the decay of muons in flight in a storage ring. This facility has the best overall performance for the discovery of $\mathrm{CP}$ violation in the neutrino sector out of all possible future facilities [1]. The main goal of the International Design Study for a Neutrino Factory (IDS-NF) is to provide a Reference Design Report (RDR) for a Neutrino Factory and associated detectors by 2013. An Interim Design Report (IDR) [2] was recently published. A schematic of the accelerator facility can be found in Figure 1.

\section{Proton Driver and Target}

The proton driver includes a pulsed proton beam with average beam power of $4 \mathrm{MW}$, a repetition rate of $50 \mathrm{~Hz}$, with three bunches per train in $240 \mu \mathrm{s}, 1-3 \mathrm{~ns}$ proton bunch length and energy between 5 and $15 \mathrm{GeV}$ (with a preferred proton energy around $8 \mathrm{GeV}$ ). The beam radius should be $1.2 \mathrm{~mm}$ (RMS), with a geometric emittance less than $5 \mu \mathrm{m}$ and a $\beta^{*}$ at the target of greater than $30 \mathrm{~cm}$. Examples of proton drivers could be the Project X LINAC at Fermilab, the SPL at CERN or a Fixed Field Alteranting Gradient (FFAG) at a green field site. The baseline target consists of a liquid mercury jet inside a $20 \mathrm{~T}$ capture solenoid field (Figure 2). The MERIT experiment [3] has provided a proof-of-principle of the mercury jet target.
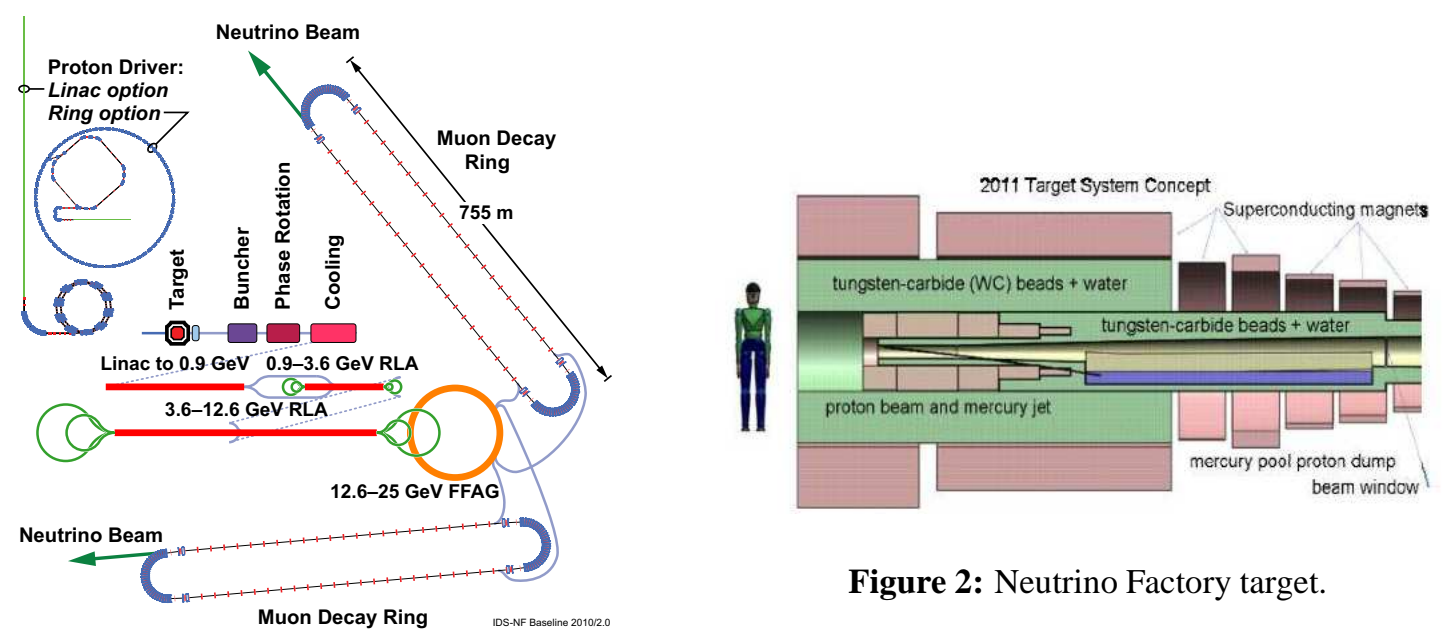

Figure 2: Neutrino Factory target.

Figure 1: Schematic drawing of the IDS-

NF accelerator complex.

\section{Muon Front End}

The Neutrino Factory muon front end consists of a pion decay channel and longitudinal drift, followed by an adiabatic buncher, phase-rotation system, and ionisation cooling channel. Downstream of the target solenoid, the magnetic field is adiabatically reduced from $20 \mathrm{~T}$ to $1.5 \mathrm{~T}$ over a distance of $15 \mathrm{~m}$. The pions then drift longitudinally over $57.7 \mathrm{~m}$ and decay to the daughter muons. The drift channel is followed by a buncher section that uses RF cavities of decreasing frequency ( 319.6 to $233.6 \mathrm{MHz}$ ) to form the muon beam into a train of bunches and a $42 \mathrm{~m}$ phaseenergy rotating section that decelerates the leading high-energy bunches and accelerates the late 
low energy bunches, so that each bunch has the same mean energy. The baseline cooling channel design consists of a sequence of identical $1.5 \mathrm{~m}$ long cells, with each cell containing two $0.5 \mathrm{~m}$ long 201.25 MHz RF cavities, two solenoid coils with opposite polarity (maximum field of $2.8 \mathrm{~T}$ providing transverse focusing with $\beta_{\perp}=0.8 \mathrm{~m}$ ), $1.1 \mathrm{~cm}$ thick $\mathrm{LiH}$ absorber discs at the ends of each cavity (four per cell) and a $0.25 \mathrm{~m}$ spacing between cavities (Figure 3). The total length of the cooling section is $75 \mathrm{~m}$ (50 cells). The cooling channel is expected to reduce the rms transverse normalised emittance from $\varepsilon_{N}=0.018 \mathrm{~m}$ to $\varepsilon_{N}=0.0075 \mathrm{~m}$ (Figure 4). The Muon Ionisation Cooling Experiment (MICE) will test one cell of the muon cooling channel [4].

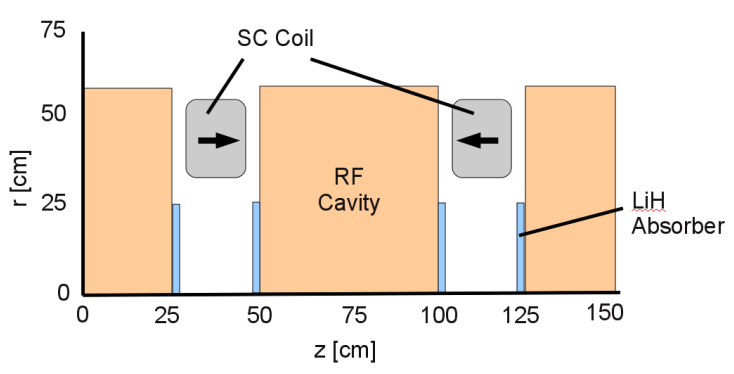

Figure 3: Baseline design of muon cooling lattice.

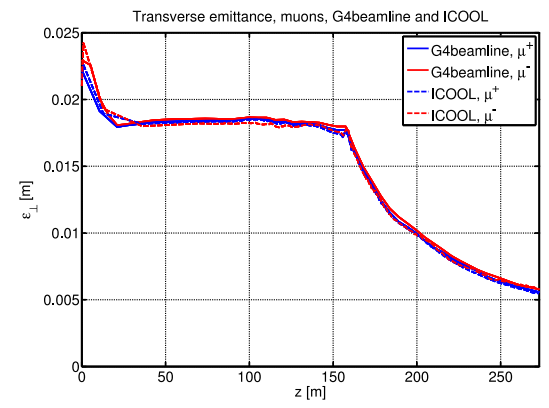

Figure 4: Performance of the cooling channel.

\section{Acceleration System, Decay Ring and Conclusions}

The acceleration proceeds in four stages: a $146 \mathrm{~m}$ LINAC that accelerates from $0.24 \mathrm{GeV}$ to $0.9 \mathrm{GeV}$, a first Recirculating Linear Accelerator (RLA) of $79 \mathrm{~m}$ length that accelerates up to 3.6 GeV, a second $264 \mathrm{~m}$ RLA up to $12.6 \mathrm{GeV}$ and a linear non-scaling Fixed Field Alternating Gradient (FFAG) up to the final energy of $25 \mathrm{GeV}$. There are two decay rings based on a racetrack design, with long $(600 \mathrm{~m})$ straight sections followed by 15 cell arcs. The decay straights point to two detectors at a distance between 2500 and $5000 \mathrm{~km}$ (18 slope) and the second at a distance between 7000 and $8000 \mathrm{~km}$ (with a slope of $36^{\circ}$ ) [5]. Muons are stored in the decay rings for about 1000 turns, with a muon beam divergence of $0.1 / \gamma$.

The International Design Study for a Neutrino Factory delivered the Interim Design Report in March 2011. The IDS-NF is on target to produce a Reference Design Report, including performance and costs, by 2013. The main concepts for the accelerator systems have been defined. The main areas of work are at the interfaces between components.

\section{References}

[1] F.J.P. Soler, "Neutrino oscillation physics with a Neutrino Factory”, PoS(EPS-HEP2011), 105.

[2] S. Choubey, et al., International Design Study for the Neutrino Factory, Interim Design Report, IDS-NF-20, https://www.ids-nf.org/wiki/FrontPage/Documentation/IDR.

[3] I. Efthymiopoulos "MERIT - The High Intensity Liquid Mercury Target Experiment at the CERN PS”, IEEE Nucl.Sci.Symp.Conf.Rec.1:3302-3305,2008.

[4] International Muon Ionization Cooling Experiment, http://mice.iit.edu/

[5] F.J.P. Soler, "Detectors for leptonic CP violation at the Neutrino Factory”, PoS(EPS-HEP2011), 394. 